\title{
ULTRACYTOCHEMICAL STUDY OF FATTY ACID ABSORPTION IN THE RAT SMALL INTESTINE BY PERFORMIC ACID-PHOSPHOTUNGSTIC ACID (PFP) REACTION
}

\author{
Midori OHTSUKI And Atsuko FUJIOKA \\ Department of Anatomy, Kinki University School of Medicine, Sayama, Osaka 589
}

Received for publication May 6, 1987 and in revised form June 30, 1987

\begin{abstract}
The early events of fatty acid absorption in the small intestine were ultracytochemically investigated by the PFP reaction, which stained unsaturated lipids. Five min after administration of the unsaturated long chain fatty acid, docosahexaenoic acid (C22:6), ordinary electron staining did not cause any morphological changes in the apical cytoplasm except for the electron dense droplets which appeared in the ER below the terminal web, but a PFP positive particulate or amorphous substance appeared in the microvilli, the terminal web and around the ER below the terminal web. It seemed to be formed by aggregation of small particles. Sometimes the particles appeared separately. The PFP positive substances did not seem to be enclosed by a membrane, and could be free fatty acid diffusing down to the ER. Fatty acid may penetrate the microvillus membranes in the form of a small particle, diffuse down the microvilli to the terminal web and be transformed to droplets by enzyme at the ER.
\end{abstract}

Dietary fat is absorbed at intestinal absorptive cells. Two different ideas on the fat absorption in the intestinal absorptive cells have been proposed ulrastructurally. One is the pinocytic mechanism $(12,13)$ and another is the diffusion mechanism across the microvillus membranes and to the teminal web $(1,9,14,15)$. Biochemical $(5,6)$ and physicochemical $(3,4,7)$ investigations seem to support the latter as the primary mechanism of fat absorption. The first prominent morphological alteration in the cells after fat intake was the appearance of electron dense droplets in the endoplasmic reticulum (ER). Although some workers proposed diffusing micelles in the microvilli $(9,14)$ some have found no morphological changes in either the microvilli or the terminal web $(1,15)$. The difficulty in visualizing micelles in the superficial cytoplasm may be due to the small size of absorbed substance or due to their unstainability by electron staining.

The PFP reaction, which has been reported to stain unsaturated lipids (2), was used to visualize the initial events of fatty acid absorption in the superficial cytoplasm of intestinal absorptive cells.

\section{MATERIALS AND METHODS}

Male Wistar rats ( 5 weeks) were starved for 2 days during which water intake was allowed. Animals were lightly anesthetized by ether and fed $1.5 \mathrm{ml}$ of fatty acid solu- 
tion, $2 \mathrm{mM}$ sodium taurodeoxycholate or $0.9 \%$ saline by oral intubation into the stomach. The fatty acid solution consisted of $7.5 \mathrm{mg}$ of 4, 7, 10, 13, 16, 19-docosahexaenoic acid (C22:6) (P-L Biochemical, Inc.) emulsified in $1.5 \mathrm{ml}$ of $2 \mathrm{mM}$ sodium taurodeoxycholate. After 5-30 min, the upper jejunum was immersed in an aldehyde mixture overnight at $0-4^{\circ} \mathrm{C}$. After washing in $0.1 \mathrm{M}$ cacodylate buffer containing $8 \%$ sucrose, $\mathrm{pH} 7.2$, for $1-2 \mathrm{hr}$ at $0-4^{\circ} \mathrm{C}$, the specimens were post-fixed in buffered osmium tetroxide for $1.5 \mathrm{hr}$ at $0-4^{\circ} \mathrm{C}$, dehydrated through a graded ethanol series, passed through propylene oxide and embedded in epon. Prior to observation under an HU-12 electron microscope, ultrathin sections were stained with either uranyl acetate followed by lead citrate or PFP. In the case of the latter, ultrathin sections mounted on a gold mesh were oxidized with freshly prepared $4.7 \%$ performic acid (10) or a diluted one with 1 part of distilled water for $30 \mathrm{~min}$ at $0-4^{\circ} \mathrm{C}$ and then treated with $1 \%$ phosphotungstic acid in distilled water for $30 \mathrm{~min}$ at $20-25^{\circ} \mathrm{C}$ (2).

\section{RESULTS}

The electron dense droplets appeared in the ER below the terminal web from the ordinary electron stained specimens at $5 \mathrm{~min}$ after fatty acid administration. There was no morphological change showing the absorption in the microvilli and the terminal web (Fig. 1). Then many droplets appeared in the Golgi cisternae and lateral intercellular spaces (not shown). Thus the pathway of docosahexaenoic acid intake seemed to coincide with that of long chain triglycerides reported previously $(1,11)$.

The glycocalyx, apical plasma membrane and apical vesicles in the apical portion of intestinal absorptive cells from $\mathrm{NaCl}$ or sodium taurodeoxycholate fed rats were PFP-positive (Fig. 2). There was no notable structure except for the apical vesicles in the superficial cytoplasm.

In the case of fatty acid administration, PFP positive droplets appeared in the cytoplasm below the terminal web at 5 min (Fig. 3). The membranes surrounding them were not identified because the PFP reaction did not stain the intracellular membranes. Judging from their location and their profile, however, they corresponded to the electron dense droplets which appeared in the ER by electron staining. In addition, a PFP positive substance which was morphologically distinguished from the droplets was observed. It appeared to be a particulate or amorphous clump (Figs. 37). Sometimes there were particles distributing individually (Figs. 4, 7). The particles appeared to be aggregated with each other to form clumps. Membranes surrounding them were not recognized. They appeared not only near the PFP-positive droplets in the superficial cytoplasm below the terminal web (Fig. 7) but also in the apical (Fig. 4) and the basal portion (Fig. 5) of the microvilli and the terminal web

FIG. 1. Apical portion of an intestinal absorptive cell. Doubly stained with uranyl acetate and lead citrate. Electron dense droplets (D) are seen in the s-ER 5 min after fatty acid administration, but no special structure is seen in either the microvilli (Mv) or the terminal web (TW). $\quad \times 47,000$

FIG. 2. Five min after saline administration. Stained with PFP. Glycocalyx (asterisk), membranes of microvilli (Mv) and apical vesicles (AV) are PFP-positive. $\quad \times 35,000$

FIGS. 3-7. Five min after fatty acid administration. Stained with PFP.

FIG. 3. Particulate or amorphous substance (arrows) in the microvilli (Mv) and the terminal web (TW) and droplets (D) in the cytoplasm below the terminal web are observed. AV: apical vesicle. $\times 25,000$ 

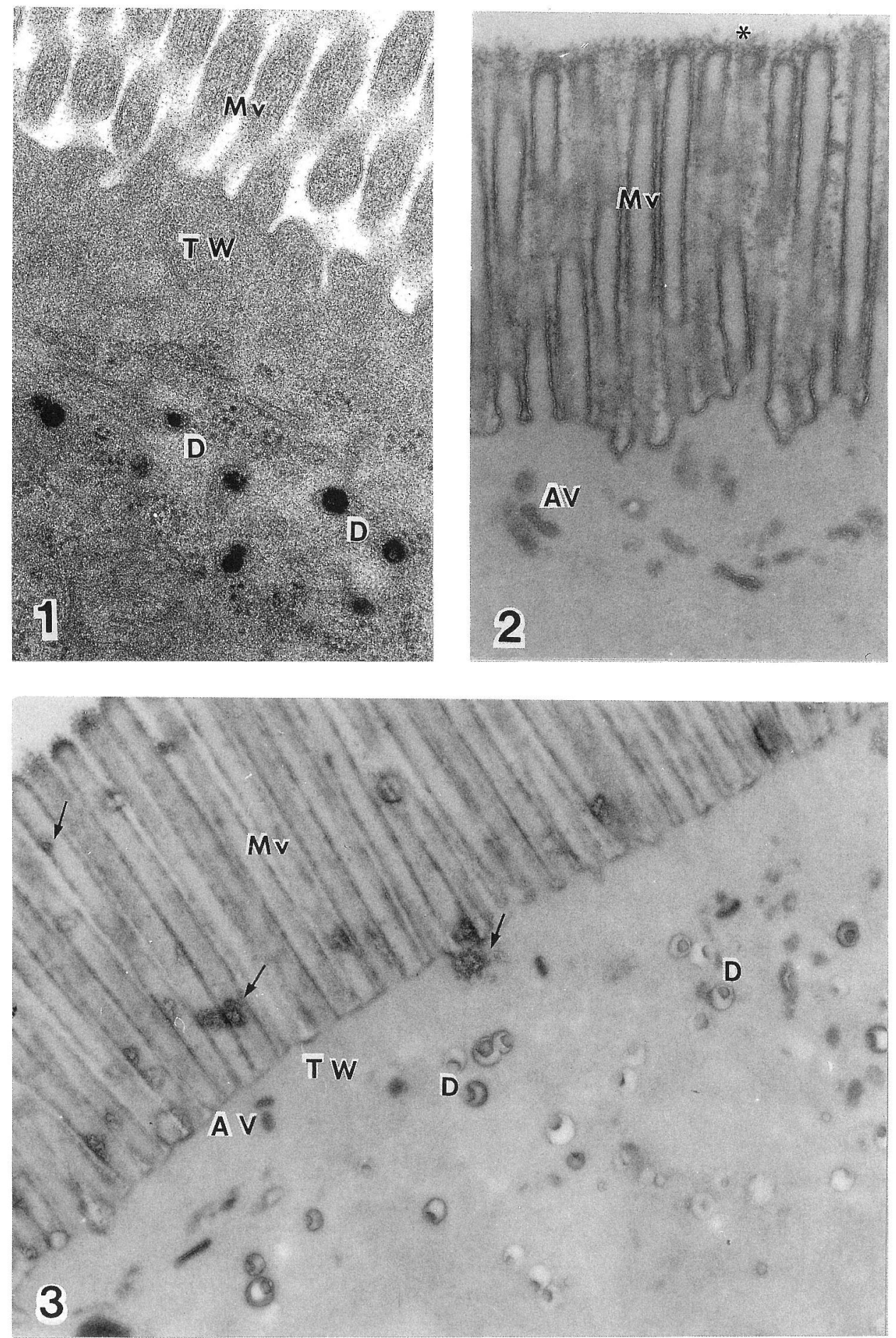

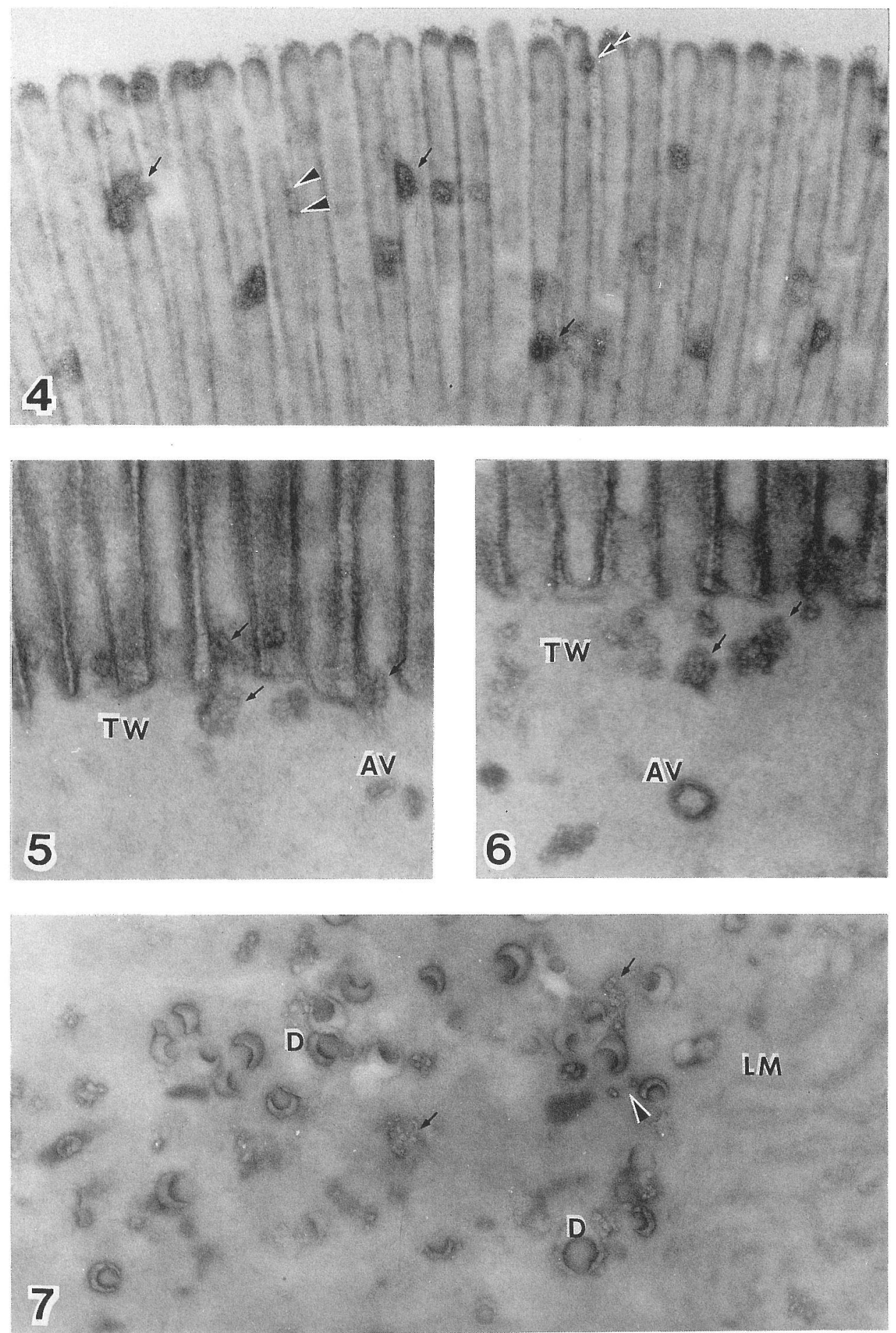
(Figs. 5, 6) where electron staining caused no morphological change even after fatty acid administration. Some particles seemed to be penetrating the microvillus membrane (Fig. 4) and some were very close to droplets in the ER (Fig. 7). These PFPpositive substances did not appear so frequently as droplets and were not seen around the Golgi apparatus even after 30 min of feeding. Little PFP positive substance was found in the intestinal lumen and the intermicrovillus space (Figs. 3, 4). After fatty acid administration, no increase in pinocytic vesicles or apical vesicles were recognized (compare Fig. 2 with Fig. 3).

\section{DISCUSSION}

We examined the early events of fatty acid absorption with docosahexaenoic acid and PFP. The former is an unsaturated long chain fatty acid with six double bonds (C22:6) and the latter is a staining method which has been proposed to stain unsaturated lipids (2). Thus docosahexaenoic acid which has as many as six double bonds is expected to be stained by PFP.

The PFP reaction revealed particles and a particulate or amorphous substance in the superficial cytoplasm of the intestinal absorptive cell of the fatty acid administered rat. As such a substance did not appear in the cells from the $\mathrm{NaCl}$ or taurodeoxycholate-fed rat, it seems to relate to fatty acid absorption. The PFP-positive substances would be the absorbed fatty acid. Some workers $(1,11,15)$ including ourselves failed to visualize fatty acids in the superficial cytoplasm by electron staining. This may be due to unstainability rather than to their small size. The fatty acid would not be visualized by the electron staining until it is transformed by enzymes at the ER (1). We can say that the PFP reaction enabled us to stain out the fatty acid being absorbed.

The PFP positive substance seemed to be in free form without an envelope, because no membranous structure except for the apical vesicles was found in the microvilli or the teminal web by electron staining. The free fatty acid without the envelope would be diffusing the microvilli and the terminal web to the underlining ER. Now it is evident ultracytochemically that fatty acid is transported to the ER by diffusion, as many workers $(1,3-7,15)$ have suggested. It may penetrate the microvillus membranes in the form of a small particle. Then the particles appear to diffuse down forming clumps and to arrive at the ER. However, the possibility can not be excluded that rearrangement of particles occurs to form clumps during electron microscopic preparation, because the particles are distributed in free form without an envelope. In any case, fatty acid would diffuse showing a particulate or amorphous profile and be transformed to the droplets by enzymes at the ER. The substance may appear less frequently than the droplets because it is easy for a freely distributing

FIG. 4. Apical portion of microvilli. A PFP positive particulate or amorphous substance forming clumps (arrows) is seen. Sometimes particles (arrow heads) and a small amorphous substance (double arrow head) appeared to be penetrating the microvillus membrane. $\times 35,000$

FIGS. 5, 6. PFP-positive particulate or amorphous substance (arrows) in basal portion of microvilli and the terminal web (TW). It appeared to be formed by the aggregation of particles. AV: apical vesicle. $\times 49,000$

Fig. 7. The cytoplasm below the terminal web. Particles (arrow head), particulate clumps (arrows) and droplets (D) are seen. LM: lateral membrane. $\quad \times 47,000$ 
substance to flow away during the electron microscopic preparation and some small substance dissolved out during dehydration (8).

The administered fatty acids were observed to follow a pathway similar to the absorption of dietary fat $(1,9)$. The initial events of long chain fatty acid absorption discussed above may be a model of fat absorption because dietary fat is absorbed after hydrolization into long chain fatty acid and monoglyceride $(3,4)$.

\section{REFERENCES}

1. Cardell, R. R., Badenhausen, S. and Porter, K. R.: Intestinal triglyceride absorption in the rat. An electron microscopical study. J. Cell Biol. 34; 123-155, 1967.

2. Fujioka, A. and Ogawa, K.: Ultracytochemistry of the cell membrane. II. The application of phosphotungstic acid staining to microvillus membranes of the cat small intestine. Acta histochem. cytochem. 12; 97-119, 1979.

3. Hofmann, A. F. and Borgstrom, B.: Physico-chemical state of lipids in intestinal content during their digestion and absorptin. Fed. Proc. 21; 43-50, 1962.

4. Hofmann, A. F.: A physicochemical approach to the intraluminal phase of fat absorption. Gastroenterology 50; 56-64, 1966.

5. Isselbacher, K. J.: Metabolism and transport of lipid by intestinal mucosa. Fed. Proc. 24; 1622, 1965.

6. Isselbacher, K. J.: Biochemical aspects of fat absorption. Gastroenterology 50; 78-82, 1966.

7. Johnston, J. M. and Borgstrom, B.: The intestinal absorption and metabolism of micellar solutions of lipids. Biochim. Biophys. Acta 84; 412-423, 1964.

8. Korn, E. D. and Weisman, R. A.: I. Loss of lipids during preparation of amoebae for electron microscopy. Biochim. Biophys. Acta 116; 309-316, 1966.

9. Lacy, D. and Taylor, A. B.: Fat absorption by epithelial cells of the small intestine of the rat. Am. J. Anat. 110; 155-185, 1962.

10. Lillie, R. D. and Fullmer, H. M.: Histopathologic Technic and Practical Histochemistry, 4th ed., McGraw-Hill Book Company, New York, 1976, p. 220.

11. Ohshima, Y.: An electron microscope study of the intestinal absorption of medium chain and long chain triglycerides in the rat. Arch. Histol. jap. 40; 153-169, 1977.

12. Palay, S. L. and Karlin, L. J.: An electron microscopic study of the intestinal villus. I. The fasting animal. J. Biophys. Biochem. Cytol. 5; 363-372, 1959.

13. Palay, S. L. and Karlin, L. J.: An electron microscopic study of the intestinal villus. II. The pathway of fat absorption. J. Biophys. Biochem. Cytol. 5; 373-384, 1959.

14. Rostgaard, J. and Barrnett, R. J.: Fine structural observations of the absorption of lipid particles in the small intestine of the rat. Anat. Rec. 152; 325-350, 1965.

15. Strauss, E. W.: Electron microscopic study of intestinal fat absorption in vitro from mixed micelles containing linolenic acid, monoolein, and bile salt. J. Lipid Res. 7; 307-323, 1966. 